\title{
ON AN INEQUALITY OF SEIDEL AND WALSH
}

\author{
LYNN H. LOOMIS
}

Introduction. In a recent paper ${ }^{1}$ Seidel and Walsh introduced the following concepts.

Let $R$ be a Riemann surface (configuration) lying over the $w$-plane, and let $C_{p}$ be a simply-connected region of $R$ having the following properties :

(a) $C_{p}$ contains precisely $p$ points (counted according to branchpoint multiplicity) lying over some point of the w-plane.

(b) $C_{p}$ lies over the circle $\left|w-w_{0}\right|<r$, and the boundary of $C_{p}$ lies over the circumference $\left|w-w_{0}\right|=r$.

It follows that $C_{p}$ contains precisely $p$ points lying over every point of $\left|w-w_{0}\right|<r$, and in particular, $p$ points $\bar{w}_{i}$ lying over $w_{0}$. Seidel and Walsh name such a region a p-sheeted circle with centers $\bar{w}_{i}$ and radius $r$. Given a point $\bar{w}_{0}$ of $R$, let $r_{p}$ be the radius of the largest $p$-sheeted circle in $R$ with center $\bar{w}_{0}$; if none exists, let $r_{p}=0$. We then define the radius of p-valence of $R$ at $\bar{w}_{0}, D_{p}\left(\bar{w}_{0}\right)$, as the maximum of the $r_{n}$ for $n \leqq p$.

Let $w=f(z)=a_{1} z+\cdots+a_{p} z^{p}+a_{p+1} z^{p+1}+\cdots$ be analytic in the unit circle $|z|<1$ with $|f(z)|<M$, and let the Riemann surface $R$ be the image of $|z|<1$ under $w=f(z)$. Let $\bar{w}_{0}$ be the image of $z=0$; $\bar{w}_{0}$ lies over $w=0$. Seidel and Walsh establish the following relation between the first $p$ coefficients of $f(z)$ and the radius of $p$-valence, $D_{p}\left(\bar{w}_{0}\right)$, of $R$ at $\bar{w}_{0}$.

There exist two constants, $\lambda_{p}$ depending only on $p$, and $\Lambda_{p}$ depending on $p$ and $M$, such that

$$
\lambda_{p} D_{p}\left(\bar{w}_{0}\right) \leqq \sum_{n=1}^{p}\left|a_{n}\right| \leqq \Lambda_{p}\left[D_{p}\left(\bar{w}_{0}\right)\right]^{2^{-p}}
$$

Seidel and Walsh find for $\Lambda_{p}$ the value

$$
\Lambda_{p}=24 p M^{r}, \quad r=1-2^{-p} .
$$

In this note we prove the following two statements concerning the inequalities (1).

A. The exponent $2^{-p}$ may be replaced by $1 /(p+1)$ and this exponent is the best possible $\left(\right.$ for $\left.D_{p} \rightarrow 0\right)$.

Received by the editors February 24, 1942.

${ }^{1} \mathrm{~W}$. Seidel and J. L. Walsh, On the derivatives of functions analytic in the unit circle and their radii of univalence and of p-valence, Transactions of this Society, vol. 52 (1942), pp. 129-216. 
We show, in fact, that there is a constant $K_{p}$ depending only on $p$ such that

$$
\sum_{n=1}^{p}\left|a_{n}\right| \leqq K_{p} M^{p /(p+1)}\left[D_{p}\left(\bar{w}_{0}\right)\right]^{1 /(p+1)},
$$

and that there is a class of functions actually attaining the bound $M$ for which

$$
\sum_{n=1}^{p}\left|a_{n}\right| \geqq M^{p /(p+1)}\left[\dot{D}_{p}\left(\bar{w}_{0}\right)\right]^{1 /(p+1)} .
$$

B. The coefficient $\lambda_{p}$ may be replaced by 1 , and this value of the constant is the best possible.

All proofs are based on the theorem of Rouché. We shall always suppose, without explicit statement, that $z$ is confined to the unit circle $|z|<1$.

Proof of (2). To prove (2) we assume $a_{p} \neq 0$ and let

$$
P(z)=\sum_{n=1}^{p} a_{n} z^{n}=a_{p} \prod_{n=1}^{p}\left(z-b_{n}\right),
$$

from which we have

$$
|P(z)| \leqq \sum_{n=1}^{p}\left|a_{n}\right|=C .
$$

We observe that $\left|a_{n}\right| \leqq M$, so that $|P(z)|<p M$, and therefore by the Schwarz lemma,

$$
|f(z)-P(z)|<(p+1) M|z|^{p+1}
$$

Now let

$$
\begin{aligned}
P_{1}(r) & =\left|a_{p}\right| \prod_{n=1}^{p}\left(r-\left|b_{n}\right|\right)=c_{1} r+\cdots+c_{p} r^{p} \\
C^{\prime} & =\sum_{n=1}^{p}\left|c_{n}\right|=\left|a_{p}\right| \prod_{n=1}^{p}\left(1+\left|b_{n}\right|\right) .
\end{aligned}
$$

Then by (4) and (7), $\left|P\left(r e^{i \theta}\right)\right| \geqq\left|P_{1}(r)\right|$. Also, since $c_{n}$ is the same polynomial in the $\left|b_{j}\right|$ as $a_{n}$ is in the $b_{j}$, and since only plus signs (or only minus signs) occur, we have $\left|c_{n}\right| \geqq\left|a_{n}\right|$, and $C^{\prime} \geqq C$. Together with (7) this gives

$$
\left|P\left(r e^{i \theta}\right)\right| \geqq C\left|\prod_{n=1}^{p} \frac{r-\left|b_{n}\right|}{1+\left|b_{n}\right|}\right| .
$$


Note that if $\left|b_{i}\right|>1$ we only strengthen the inequality (8) if we replace $\left|b_{i}\right|$ by 1 in the quotients occurring there. But then (8) implies that

$$
\left|P\left(r e^{i \theta}\right)\right| \geqq C 2^{-p} \prod_{n=1}^{p}\left|r-b_{n}^{\prime}\right|,
$$

where $b_{n}^{\prime}=\left|b_{n}\right|$ if $\left|b_{n}\right| \leqq 1$, and $b_{n}^{\prime}=1$ otherwise. Now choose any positive real number $x_{0}<1$. In the interval $\left(0, x_{0}\right)$ there is at least one point $r_{1}$ such that for $i \leqq p$,

$$
\left|r_{1}-b_{i}^{\prime}\right| \geqq x_{0} / 2^{p},
$$

and therefore by (9),

$$
\left|P\left(r_{1} e^{i \theta}\right)\right| \geqq C x_{0}^{p}(4 p)^{-p},
$$

and by (6) and (10)

$$
\left|P\left(r_{1} e^{i \theta}\right)\right|-\left|f\left(r_{1} e^{i \theta}\right)-P\left(r_{1} e^{i \theta}\right)\right| \geqq C x_{0}^{p}(4 p)^{-p}-x_{0}^{p+1}(p+1) M .
$$

This expression is maximum for $x_{0}=C p /(4 p)^{p}(p+1)^{2} M$, its maximum value being $C^{p+1} / M^{p} K^{p+1}$, where $K=(p+1)((p+1) / p)^{p /(p+1)}(4 p)^{p}$.

The polynomial $P(z)$ has $n$ zeros in $|z| \leqq r_{1}$ with $n \leqq p$. Therefore by Rouché's theorem, $f(z)$ assumes in $|z| \leqq r_{1}$ every value in $|w|$ $\leqq C^{p+1} / K^{p+1} M^{p}$ precisely $n$ times. Therefore $D_{p}(0) \geqq C^{p+1} / K^{p+1} M^{p}$; that is,

$$
\sum_{n=1}^{p}\left|a_{n}\right| \leqq K M^{p /(p+1)}\left[D_{p}(0)\right]^{1 /(p+1)}
$$

as was to be proved.

In the above proof we have assumed that $a_{p} \neq 0$. If $a_{p}=0$ the proof is valid for some $n \leqq p$, and $p$ can be reintroduced in the later stages, say in equation (8), where the product can be taken over $p$ factors if some of the $\left|b_{n}\right|$ are allowed to vanish.

Proof of (3). We prove (3) by considering the class of functions

$$
\begin{aligned}
f(z) & \equiv M z^{p} \frac{a-M z}{M-a z} \\
& \equiv a z^{p} \frac{1-(M z / a)}{1-(a z / M)}
\end{aligned}
$$

where $a<M$. These functions have been studied by the author in a 
previous note. ${ }^{2}$ From (11) it is clear that $|f(z)| \leqq M$ in $|z| \leqq 1$, and that the bound is attained for $z=1$. It is also evident from (11) that the minimum of $|f(z)|$ on $|z|=r<a / M$ occurs at $z=r$. Thus $|f(z)| \geqq f(r)$ for $|z|=r<a / M$. By Rouché's theorem $f(z)$ covers the circle $|w|<f(r)$ precisely $p$ times in $|z|<r$, since $f(z)=0$ has $p$ roots in this circle. By Rolle's theorem $f^{\prime}(z)$ vanishes for some $r_{0}$ between $r=0$ and $r=a / M$. Then every value of $|w| \leqq f\left(r_{0}\right)$ except $w=f\left(r_{0}\right)$ is assumed precisely $p$ times in $|z| \leqq r_{0}$, and $w=f\left(r_{0}\right)$ is assumed $p+1$ times. Therefore $D_{p}(0)=f\left(r_{0}\right)$. But by (12)

$$
D_{p}(0)=f\left(r_{0}\right)<a\left(r_{0}\right)^{p}<a(a / M)^{p}=a^{p+1} / M^{p} .
$$

Also by (12), the first term in the expansion of $f(z)$ is $a z^{p}$ so that the sum of the moduli of the first $p$ coefficients is $a$. Thus by (13)

$$
\sum_{n=1}^{p}\left|a_{n}\right|=a>M^{p /(p+1)}\left[D_{p}(0)\right]^{1 /(p+1)}
$$

which is the desired inequality (3).

The coefficient $\lambda_{p}$. We have left to prove that $\lambda_{p}$ can be taken as 1 ; in other words, that

$$
D_{p}(0) \leqq \sum_{n=1}^{p}\left|a_{n}\right|=C
$$

As before, let $P(z)=a_{1} z+\cdots+a_{p} z^{p}$, and suppose that $D_{p}(0)>C$. Then $|f(z)|=C$ defines an analytic Jordan curve $\Gamma$ in $|z|<1$ and $\Gamma$ contains the origin in its interior. On $\Gamma$ we, have $|P(z)|<C=|f(z)|$. Therefore by Rouché's theorem $f(z)-P(z)$ and $f(z)$ have the same number of zeros interior to $\Gamma$. But $f(z)$ has at most $p$ zeros inside $\Gamma$ since the image of the interior of $\Gamma$ under $f(z)$ is an $n$-sheeted circle with $n \leqq p$. And $f(z)-P(z) \equiv a_{p+1} z^{p+1}+\cdots$ has at least $p+1$ zeros interior to $\Gamma$. By this contradiction we see that $D_{p}(0) \leqq C$ as we wished to prove.

This result is trivially the best possible, for if $f(z) \equiv a z^{p}$, then $D_{p}(0)=a=C=M$.

\section{HARVARD UNIVERSITY}

${ }^{2}$ L. H. Loomis, The radius and modulus of n-valence for analytic functions whose first $n-1$ derivatives vanish at a point, this Bulletin, vol. 46 (1940), pp. 406-501.

Two problems were considered in this note. One was the problem of the present paper for the class of functions whose first non-vanishing coefficient is $a_{p}$. The second was the problem of determining for this restricted class of functions the largest circle $|z|<r$ (the radius depending on $p, M$ and $\left|a_{p}\right|$ ) in which all the functions are at most $p$-valent. The present note does not treat the corresponding problem for the more general class of functions considered here. 